\title{
A Human Fibroblast-Derived Growth Factor Preparation in the Management of a Chronic Surgical Wound in a Diabetic Patient: A Case Report
}

\section{Maria Angelo-Khattar (D) Georgia Vadarli $\mathbb{D}^{2}$}

'American Academy of Anti-Aging Medicine (A4M), Dubai, United Arab Emirates; ${ }^{2}$ Consultant Plastic Surgeon, Thessaloniki, Greece
Correspondence: Maria Angelo-Khattar American Academy of Anti-Aging Medicine, Building 39, Dubai Healthcare City, Dubai, United Arab Emirates Tel +97I506245494

Emailmkhattar@younatagroup.com

\begin{abstract}
The treatment of choice in patients with ischaemic heart disease is coronary artery bypass grafting. The procedure entails the harvesting of the great saphenous vein through a significant leg incision, which may result in infections and wounding at the incision site. Patients with diabetes mellitus pose a greater risk of developing non-healing wounds, which may significantly affect the patient's quality of life. The use of anti-inflammatory factors and other chemokines derived from cultured human fibroblasts may represent a useful therapeutic approach for the management of surgical wounds in patients with the greatest probability of being wound healing compromised. This case study describes the treatment of a non-healing surgical tibial wound in a male diabetic patient treated with a preparation of human anti-inflammatory interleukins cytokines, and growth factors. The treatment resulted in swift recovery, significant pain reduction and complete wound closure with minimal scarring.
\end{abstract}

Keywords: chronic wound, growth factors, cytokines, coronary artery bypass graft

\section{Introduction}

The surgical treatment of choice for ischaemic heart disease is Coronary Artery Bypass Grafting (CABG). ${ }^{1}$ The Great Saphenous Vein (GSV) is the most frequently used conduit for CABG, and the technique for harvesting the GSV entails a long open skin incision, usually comprising the entire leg. The vast proportion of patients experience leg wounds as opposed to sternal wounds. The rate of great saphenous vein harvest site infections (GSVHSI) is reported to be between $1 \%$ and $24 \%{ }^{2}$ Risk factors associated with GSVHSI include peripheral vascular disease, diabetes mellitus, smoking, obesity, and chronic renal failure. ${ }^{3}$ It is well a recognized fact that one of the most significant challenges to wound healing is infection, leading to a poor prognosis and increased morbidity. ${ }^{4}$ Hence, although rare, complications arising from the great saphenous vein harvest site may require surgical debridement leading to a delay in recovery and may negatively impact the patient.

Wound healing is a physiological process that occurs through three programmed phases: inflammation, proliferation, and matrix remodelling. Effective wound healing entails that all three phases occur in an orderly fashion. Several factors can 
interfere with this process and may lead to impaired wound healing. One of the factors known to negatively impact normal wound healing is diabetes mellitus. The condition, associated with hyperglycaemia, chronic inflammation, and dysfunction of the microcirculation, may impair normal wound healing and result in chronic wounds. ${ }^{4}$ When diabetic patients develop wounds, they are at high risk for severe complications including infections and amputations. The pathophysiological mechanism responsible for impaired wound healing in diabetes is complex and involves immune, biochemical, vascular and neuropathic mechanism leading to altered soft tissue repair. Conventional treatment of chronic wounds in diabetic patients involves scrupulous wound care and stringent glucose control, despite which diabetic wounds show a poor prognosis.

Since chronic wounds are associated with tissue necrosis and death, the therapeutic use of anti-inflammatory growth factors may be of benefit in modulating the wound-healing response. Growth factors, interleukins and cytokines have physiological roles in the three phases of normal wound-healing, namely inflammation, proliferation, and matrix remodelling. Plateletderived growth factor (PDGF) activates cells of mesenchymal origin and stimulates the formation of new blood vessels. Epidermal growth factor (EGF) stimulates the regeneration of the epidermis and fibroblast growth factor (FGF) stimulates fibroblasts to synthesise collagen, elastin and glycosaminoglycans. The multitude of transforming growth factors (TGF) also stimulate the formation of extracellular matrix as well as inhibit its degradation. ${ }^{5,6}$ Furthermore, levels of PDGF, TGF-Beta, FGF and EGF have been found to be decreased in chronic wounds.

The topical application of anti-inflammatory growth factors, interleukins and cytokines may prove to be effective in the regeneration of non-healing wounds in diabetic patients.

\section{Patient and Methodology Case Presentation}

A 65-year-old man with a history of type 2 diabetes underwent Aortic Valve Replacement (AVR) and Coronary Artery Bypass graft with Saphenous vein graft (CABG). In the first postoperative week, the patient presented with oedema and erythema at the incision site on the left tibia (venous graft site).

The patient has provided the authors informed consent to publish the details of the case and all relevant images.

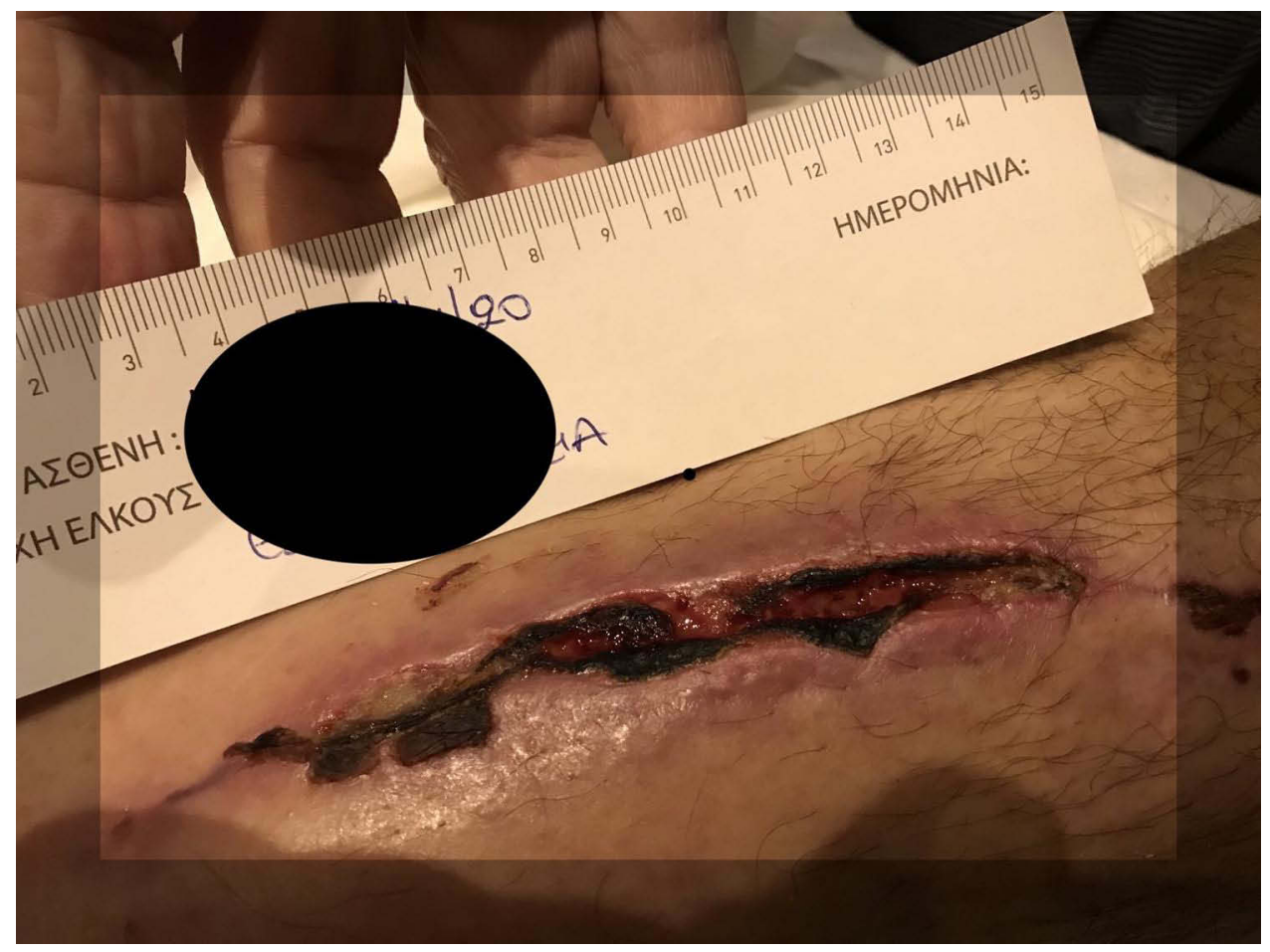

Figure I Wound on medial left tibia post CABG surgery. 
Institutional approval was not required to publish this case.

\section{Case History}

The patient underwent Aortic Valve Replacement (AVR) and Coronary Artery Bypass Graft with Saphenous vein (CABG) on October 15, 2020, at a private clinic in Athens. During the first postoperative week, he presented with oedema and erythema on the surface of the incision of the left tibia (venous graft site) and a partial opening of the wound with necrotic eschar. Two weeks after the operation, on October 30, 2020, he returned to Thessaloniki, his place of residence. Two weeks later, on November 14th 2020, he consulted a private vascular surgeon to be treated for the open wound in his left tibia (Figure 1).

Despite the regular dressing changes, the wound remained open and caused him mild pain when walking; therefore, on December 6, 2020, he sent a photo of the wound to his cardiothoracic surgeon who referred him to a plastic surgeon (Figure 2).

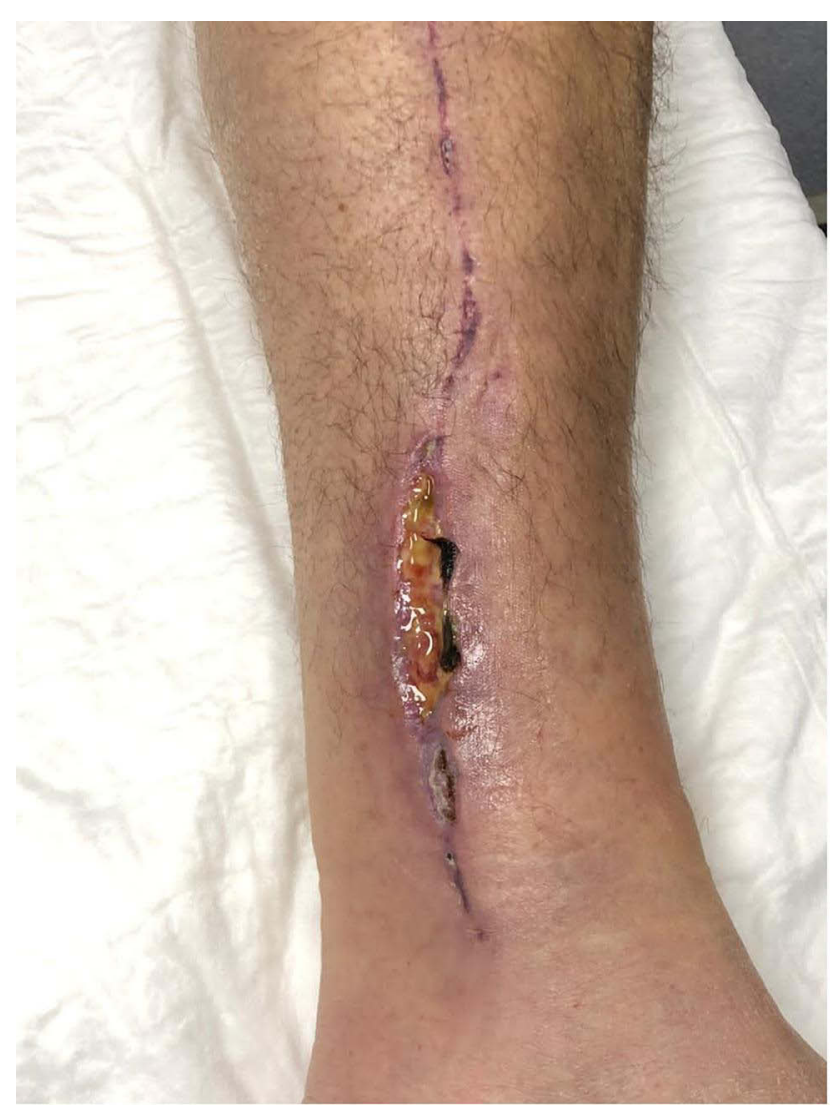

Figure 2 Day I: Wound pre-debridement.

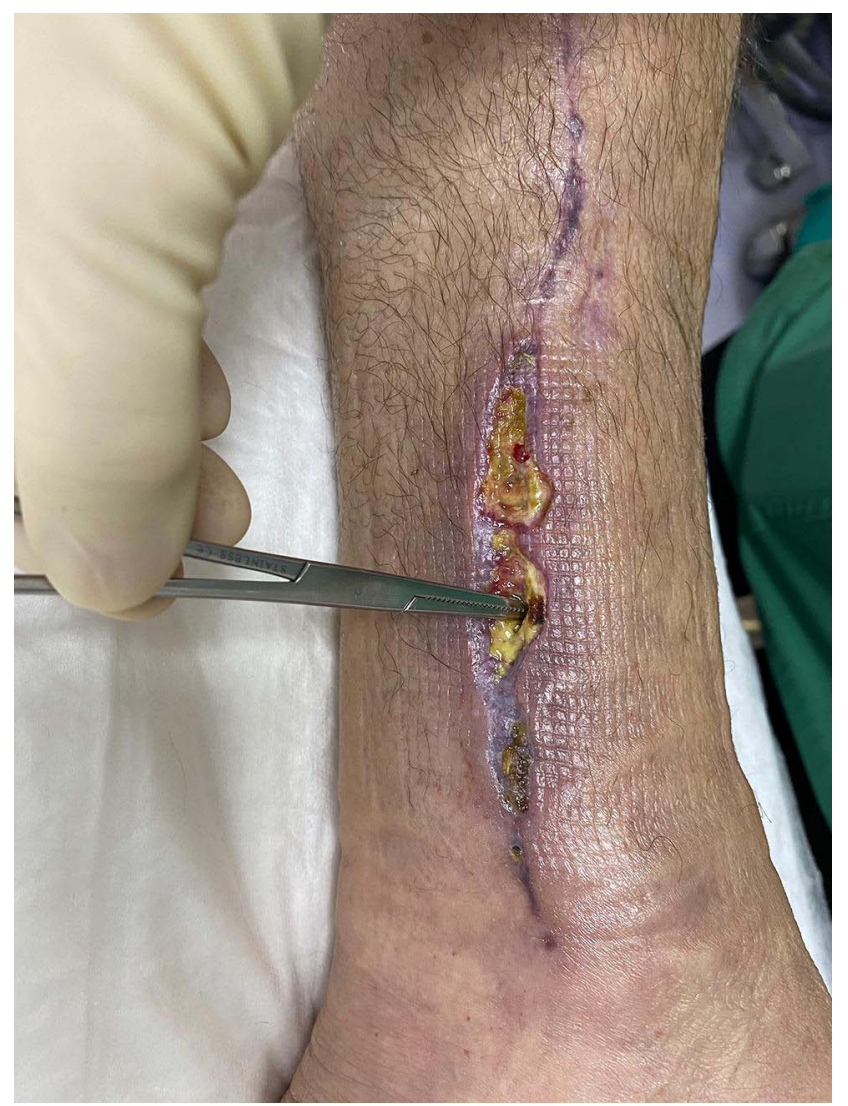

Figure 3 Day I: Post debridement and application of $2 \mathrm{~mL}$ of human growth factor preparation.

\section{Physical Examination}

The patient was referred to the plastic surgeon, Dr. Vadarli (author) on December 7, 2020. Upon examination, Dr. Vadarli found an open wound (length $=7.5 \mathrm{~cm}$ and width $=0.5 \mathrm{~cm}$ to $1.5 \mathrm{~cm}$ ) on the medial surface of the left

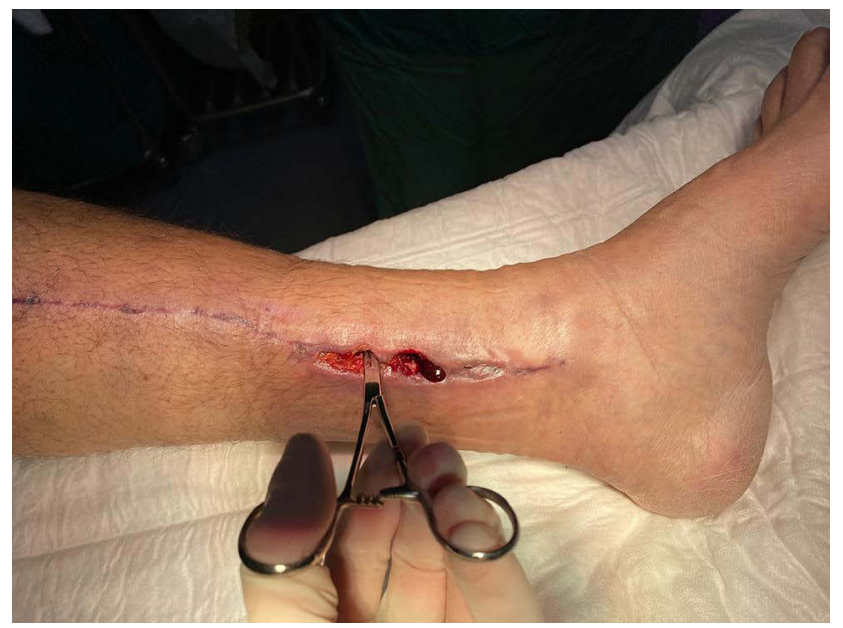

Figure 4 Day 4: Application of I $\mathrm{mL}$ of human growth factor preparation. 
tibia with a small necrotic eschar on one edge of the wound and a subcutaneous cavity $1.5 \mathrm{~cm}$ deep at two points on the outer edge of the wound (Figure 2). In addition, the patient complained of mild pain when walking.

The wound was surgically cleansed, and fluid was obtained for culture. The culture was positive for Escherichia coli and Klebsiella ozaenae. According to the antibiogram, both microbes were sensitive to Ciprofloxacin.

\section{Therapeutic Plan}

Two days after the surgical cleansing of the wound and the sampling of fluid for culture, the patient was treated at Dr. Vadarli's outpatient clinic. The tibial wound was cleansed and treated with the first application of a synergistic anti-inflammatory preparation of human fibroblast-derived cytokines, interleukins and growth factors (AQ Recovery Serum, AQ Skin Solutions, Irvine, California, USA). On the same day, the patient was started on Ciprofloxacin 500mg film-coated tablets, twice a day for two weeks, as the laboratory tests found the microorganisms to be sensitive to the antibiotic.

The wound treatment plan included the following:

The wound was cleansed with Betadine solution, irrigated with hydrogen peroxide and followed with $0.9 \%$ normal saline. AQ Recovery Serum was applied both inside and on the surface of the wound. The area was covered with sterile gauze, and dressed with elastic bandage. The dressing changes were scheduled to take place every third day.

In the initial treatment, $2 \mathrm{~mL}$ of the synergistic growth factor preparation was used (Figure 3).

During the second treatment (Figure 4) only $1 \mathrm{~mL}$ of synergistic growth factor preparation was used.

However, subsequent treatments, on days 10, 13, 16, 20 and 23 were done (Figure 5) with $2 \mathrm{~mL}$ of the growth
A

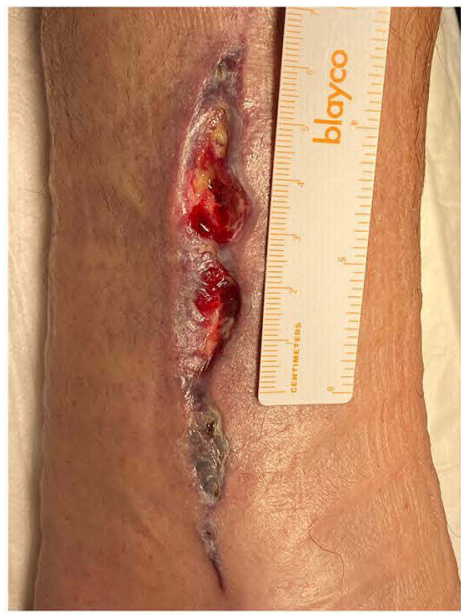

D

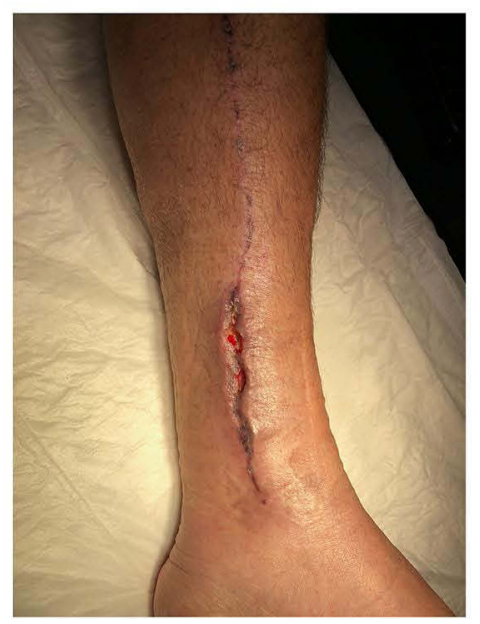

B

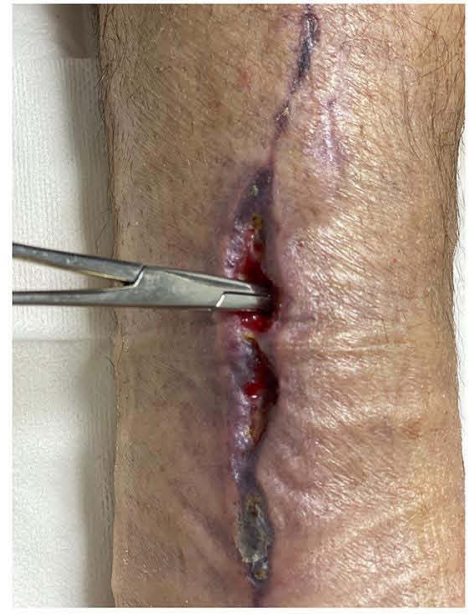

E

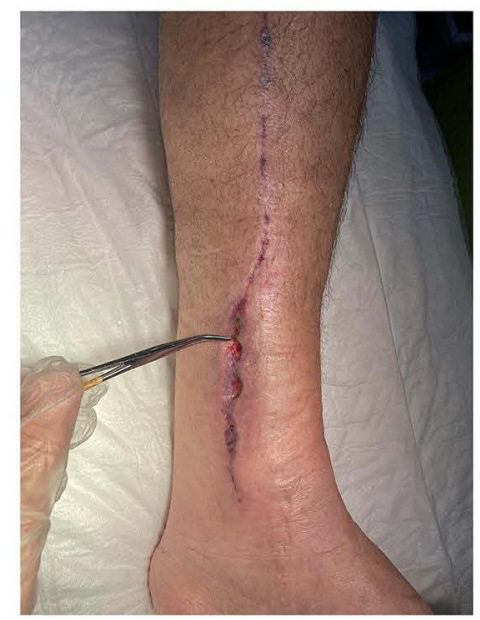

C

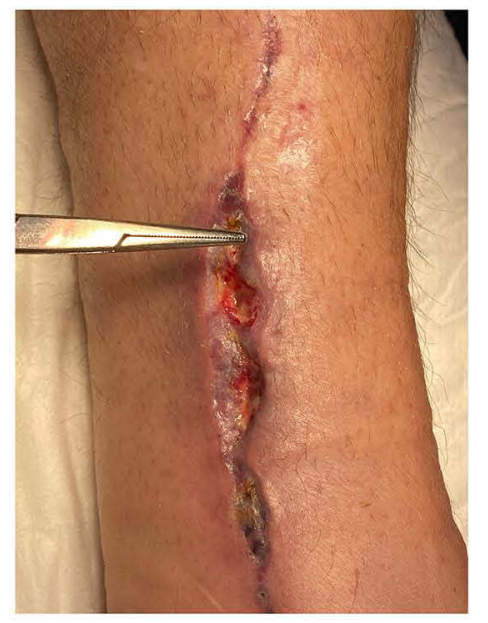

$\mathbf{F}$

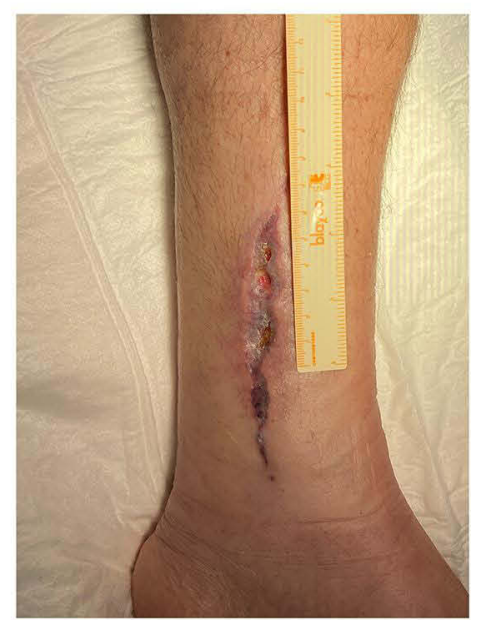

Figure 5 Representative images of wound during healing process. (A) Day 10, (B) Day 13, (C) Day 16, (D) Day 20, (E) Day 23, (F) Day 27. 
factor complex, as it appeared that the $1 \mathrm{~mL}$ dose did not have a satisfactory healing outcome. The total amount of AQ recovery serum used was $15 \mathrm{cc}$.

The preparation derived from cultured human fibroblasts contains Transforming Growth Factors (TGF) Beta-1, 2 and 3, Platelet-Derived Growth Factor (PDGF), Granulocyte Monocyte Colony Stimulating Factor (GMCSF), and Interleukins (IL) 3, 6, 7 and 8.

\section{Results}

The outcome of the human fibroblast-derived growth factor treatment of the non-healing wound created by GSVHSI in the diabetic patient was positive. The open and deep wound in the left tibia responded to the treatment relatively quickly, within 27 days of the initial application of the human fibroblast-derived growth factor serum.

The patient had no complications, and the pain was almost negligible three days following the first application of the serum. The total amount of AQ Recovery Serum used was $15 \mathrm{cc}$ (approximately four vials). Follow-up at three months showed total wound closure with only a minimal erythematous scar (Figure 6).

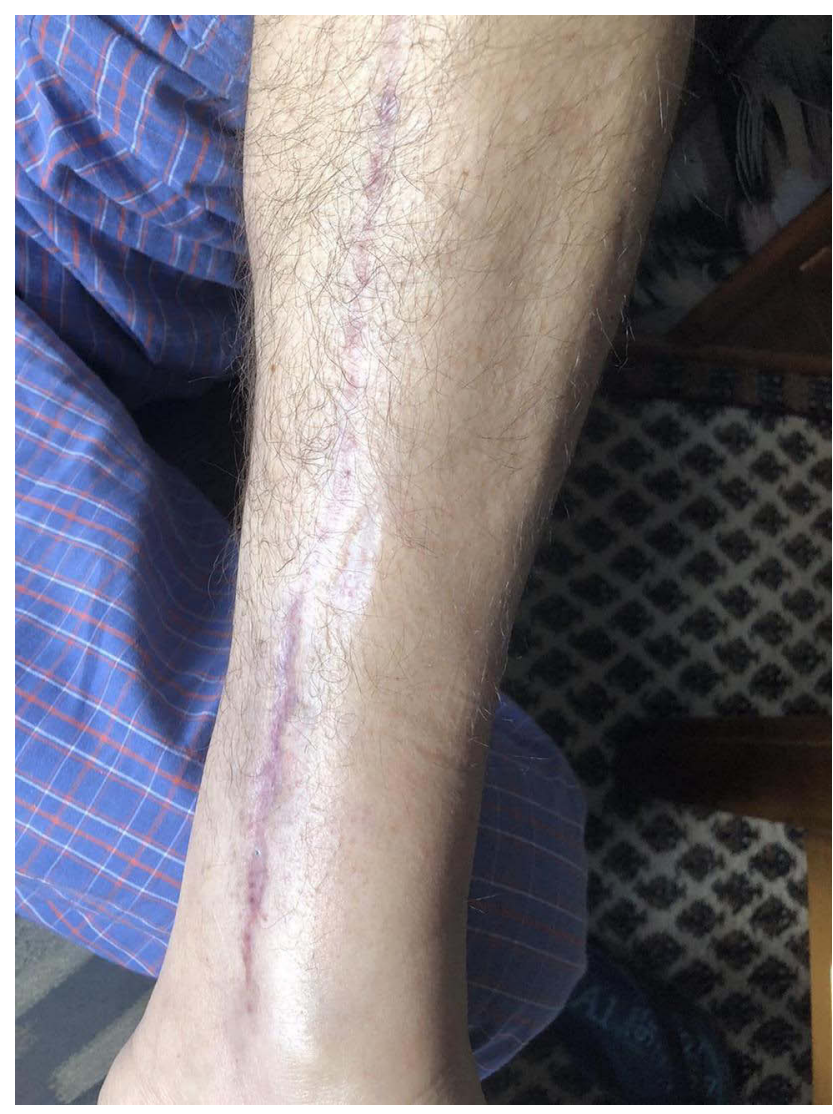

Figure $\mathbf{6}$ Three months post initiation of human growth factor treatment.

\section{Discussion}

The mainstay of ischaemic heart disease treatment is coronary bypass surgery, which entails the harvesting of the greater saphenous vein via long open incisions of the entire leg. Occasionally, the great saphenous vein harvest site infections may lead to wounds, especially in susceptible individuals such as patients with a high BMI, diabetes mellitus and smokers. ${ }^{7,8}$ Non-healing wounds may arise, requiring debridement and diligent wound management.

Normal wound closure is contingent upon an orderly physiological wound healing pattern involving the three phases of inflammation, proliferation, and matrix remodelling. Each of these phases of wound healing are dependent upon a cascade of interleukins, growth factors, and cytokines. Hence, one would infer that the enhancement of these regenerative protein titres by the topical application may optimize the wound healing process. Recently, the therapeutic value of this human fibroblast-derived growth factors preparation (AQ Recovery Serum, AQ Skin Solutions, Irvine, California, USA) has been shown in the treatment of a grade 3 radiodermatitis burn. The burn wound was shown to heal within 21 days, without pain or discomfort and negligible scarring. ${ }^{9}$

The case of the 65-year-old male patient with type 2 diabetes is noteworthy in that the non-healing wound resolved within 27 days without pain or discomfort and the scar was minimal at three months.

\section{Conclusion}

Chronic, non-healing surgical wounds in diabetic patients may be psychologically and physically disturbing. Therefore, the timely treatment of these wounds by the stimulation of an organised wound-healing response may allow for a timely patient recuperation and resolution of the scar with minimal scarring.

The treatment of a 65 -year-old male patient with type 2 diabetes with a topical preparation of human fibroblastderived anti-inflammatory cytokines, interleukins, and growth factors showed rapid recovery with a substantial reduction in pain and minimal scarring, seen at three months post-treatment.

\section{Ethics and Consent}

The patient has given informed consent to publish details of his case and to publish all the accompanying images. No institutional approval was necessary for the publication of this case. 


\section{Disclosure}

The authors report no conflicts of interest in this work.

\section{References}

1. Athanasiou T, Aziz O, Skapinakis P, et al. Leg wound infection after coronary artery by- pass grafting: a meta-analysis comparing minimally invasive versus conventional vein harvesting. Ann Thorac Surg. 2003;76(6):2141-2146. doi:10.1016/S0003-4975(03)01435-8

2. Siddiqi MS. Saphenous vein harvest wound complications: risk factors, identification, prevention and management. Chronic Wound Care Manag Res. 2016;3:147-156. doi:10.2147/CWCMR.S82601

3. Hassoun-Kheir N, Hasid I, Bozhko M, et al. Risk factors for limb surgical site infection following coronary artery bypass graft using open great saphenous vein harvesting: a retrospective cohort study. Interact Cardiovasc Thorac Surg. 2018;27:530-535. doi:10.1093/ icvts/ivy137
4. Rahim K, Saleha S, Zhu X, et al. Bacterial contribution in chronicity of wounds. Microb Ecol. 2017;73:710-721. doi:10.1007/s00248-0160867-9

5. Cooper DM, Yu EZ, Hennessey P, Ko F, Robson MC. Determination of endogenous cytokines in chronic wounds. Ann Surg. 1994;219:688-692. doi:10.1097/00000658-199406000-00012

6. Pierce GF, Tarpley JE, Tseng J, et al. Detection of platelet-derived growth factor (PDGF)-AA in actively healing human wounds treated with recombinant PDGF-BB and absence of PDGF in chronic non-healing wounds. J Clin Investig. 1995;96:1336. doi:10.1172/ JCI118169

7. Okonkwo UA, DiPietro LA. Diabetes and wound angiogenesis. Int J Mol Sci. 2017;18:1419. doi:10.3390/ijms18071419

8. Guo S, Dipietro LA. Factors affecting wound healing. J Dent Res. 2010;89:219-229. doi:10.1177/0022034509359125

9. Vadarli G, Angelo-Khattar M. A fibroblast-derived human growth factor preparation for the management of acute radiodermatitis: a case report. Int Med Case Rep J. 2020;13:691-696. doi:10.2147/ IMCRJ.S287033

\section{Publish your work in this journal}

The International Medical Case Reports Journal is an international, peer-reviewed open-access journal publishing original case reports from all medical specialties. Previously unpublished medical posters are also accepted relating to any area of clinical or preclinical science. Submissions should not normally exceed 2,000 words or 4 published pages including figures, diagrams and references. The manuscript management system is completely online and includes a very quick and fair peer-review system, which is all easy to use. Visit http://www.dovepress.com/testimonials.php to read real quotes from published authors. 